\title{
Domain characterizations of certain functions of power-bounded operators
}

by

\author{
Markus HaAse (Delft) and Yuri Tomilov (Toruń and Warszawa)
}

\begin{abstract}
We create a general framework for describing domains of functions of power-bounded operators given by power series with log-convex coefficients. This sheds new light on recent results of Assani, Derriennic, Lin and others. In particular, we resolve an open problem regarding the "one-sided ergodic Hilbert transform" formulated in a 2001 paper by Derriennic and Lin.
\end{abstract}

1. Introduction. The mean ergodic theorem for power-bounded operators $T$ on a reflexive Banach space $X$ states that

$$
P x:=\lim _{n \rightarrow \infty} \frac{1}{n+1} \sum_{k=0}^{n} T^{k} x
$$

exists in the norm of $X$ for any $x \in X$, and the limit operator $P$ is the projection onto the subspace $\operatorname{fix}(T)=\operatorname{ker}(I-T)$ of fixed points of $T$. It is known that there is no, in general, universal rate of decay in 1.1) (see e.g. [2, Theorem 4]). However, in view of the applications to probability theory, e.g. the Central Limit Theorem for Markov chains, and for a deeper understanding of ergodic issues, e.g. pointwise ergodic theorems, it is of interest to identify vectors $x$ for which the convergence in (1.1) takes place with a specified - e.g. polynomial — rate. This leads to the study of solutions to "Poisson" equations $(I-T)^{s} x=y, s \in(0,1]$, for fixed $y \in X$, and the associated series representations of these solutions.

Rates of convergence in the mean ergodic theorem and their relations to Poisson equations were treated in detail in [1, 3, 6, 7, 10, 8, 9, 11] and-from a somewhat different point of view - in [30]. In [11, basic for our considerations here, subtle results were obtained for rates of convergence of means of $\left(T^{n}\right)_{n \geq 0}$, as well as their pointwise counterparts for Dunford-Schwartz operators. In particular, as a consequence of the power series representations for

2010 Mathematics Subject Classification: Primary 47A60, 47A35; Secondary 47D03. Key words and phrases: functional calculus, power-bounded operator, domains, logarithmically convex coefficients. 
$(I-T)^{-s}, s \in(0,1)$, it was proved that the ranges of the operators $(I-T)^{s}$ "realize" the polynomial rates of decay o $\left(1 / n^{r}\right), r<s$, in the mean ergodic theorem. The case $s=1$ was treated in $[3]$. There it is also shown that the rate $\mathrm{o}(1 / n)$ in $(1.1)$ implies that $x=0$.

The family of operators $\left((I-T)^{s}\right)_{\operatorname{Re} s>0}$ forms a holomorphic semigroup of angle $\pi / 2$ and it is natural to consider its generator $G$ (defined via the Laplace transform). If the range of $I-T$ is dense in $X$ then this semigroup is strongly continuous at zero. (This is the case considered in [11.) Intuitively, one should be able to write $G=\log (I-T)$ and it is natural to ask how $G$ relates to the operator

$$
H_{T}:=\sum_{k \geq 1} \frac{T^{k}}{k}, \quad \operatorname{dom}\left(H_{T}\right):=\left\{x \in X \mid \sum_{k \geq 1} T^{k} x / k \text { exists }\right\},
$$

called the one-sided ergodic Hilbert transform. (It is not clear to us who introduced this name, but it is used in [11] and subsequent papers. The concept itself, although in a purely ergodic-theoretic context, dates back at least to [18] and has been considered many times since, e.g. in [14] and [19]. Note that the two-sided ergodic Hilbert transform has also been studied, e.g. in [4.)

It was proved in [11, Prop. 2.21] that $G$ always extends $H_{T}$ and it was asked whether the equality $G=H_{T}$ holds in general. In a number of particular situations, e.g. when $T$ is a normal contraction, this equality can be established (see [1, 10, 8]), but the general question seems to be still open.

Secondly, in [11, Prop. 2.20] it is shown that $\operatorname{ran}(I-T)^{s} \subset \operatorname{dom}\left(H_{T}\right)$ for each $0<s<1$. (Note that from the theory of holomorphic semigroups it is immediate that $\operatorname{ran}(I-T)^{s} \subset \operatorname{dom}(G)$ whenever $\operatorname{Re} s>0$.) Elements in

$$
\operatorname{dom}\left(H_{T}\right) \backslash \bigcup_{s>0} \operatorname{ran}(I-T)^{s}
$$

provide examples of non-polynomial convergence rates in the mean ergodic theorem. It was proved in [1] that for a unitary operator $T$ with $1 \in \sigma(T)$,

$$
\bigcup_{s>0} \operatorname{ran}(I-T)^{s} \neq \operatorname{dom}\left(H_{T}\right)
$$

but it was left as an open question whether this is true in general.

In the present paper we shall resolve the above-mentioned two problems in the affirmative. More precisely, we shall show that if $T$ is a power-bounded operator on a Banach space such that $I-T$ has dense range, then $H_{T}=$ $\log (I-T)$ (Theorem 6.2). If, in addition, $I-T$ is not invertible, then indeed

$$
\bigcup_{s>0} \operatorname{ran}(I-T)^{s} \neq \operatorname{dom}\left(H_{T}\right)
$$

(Theorem 6.3). Our approach is operator-theoretic and uses the theory 
of functional calculus (for power-bounded and sectorial operators, respectively), thereby avoiding technicalities and providing a conceptual and transparent treatment. Apart from answering the above-mentioned questions, our approach yields new proofs for some other results of [11], e.g. the power series characterization of the ranges $\operatorname{ran}(I-T)^{s}, 0<s<1$.

The paper is organized as follows. In Section 2 we review the construction of the functional calculus for power-bounded operators, based on the map

$$
f(z)=\sum_{k \geq 0} \alpha_{k} z^{k} \mapsto f(T):=\sum_{k \geq 0} \alpha_{k} T^{k}
$$

for $\alpha=\left(\alpha_{k}\right)_{k} \in \ell^{1}$. General functional calculus theory (as developed in [13, Chapter 1]) allows one to extend this functional calculus to more general functions/power series. The main objective of the paper will be to characterize the domain $\operatorname{dom}(f(T))$ by means of the convergence of

$$
\sum_{k \geq 0} \alpha_{k} T^{k} x
$$

when $f$ has the Taylor expansion $f(z)=\sum_{k=0}^{\infty} \alpha_{k} z^{k}$.

In Section 3 we show that for a power-bounded operator $T$ the operator $I-T$ is sectorial. Then we review the functional calculus for sectorial operators from [13] and prove that the two functional calculi are compatible. This implies that the operators $(I-T)^{s}$ and $\log (I-T)$ are unambiguously defined in either calculus, and this accounts for a greater flexibility in dealing with them. In particular, one can replace the (sometimes tedious) power series arguments from [11] by invoking general theory. Examples are the analytic semigroup property of $\left((I-T)^{s}\right)_{\operatorname{Re} s>0}$ and the identification of the generator $G$ as $\log (I-T)$.

The functional calculus reduces operator-theoretic questions to functiontheoretic ones, even if the operator is not a normal operator on a Hilbert space. In Section 4 we provide some results for "admissible" holomorphic functions on the unit disc. Our major tool is a result about power series with log-convex coefficients proved by Kaluza in 1928 [20]. Finally, in Section 5 we apply the function theory via the calculus to operators, leading to our main result, Theorem 5.6. In Section 6 we apply the main result to fractional powers and the logarithm, eventually resolving both questions above.

We would like to point out that a similar description of domains of functions of sectorial operators was given in [17], based on the so-called Hirsch calculus. This line of research, related to a continuous version of our setting, was further developed in [28].

REMARK 1.1. After the completion of this paper we learnt that the characterization of the domain of $\log (I-T)$ for power-bounded $T$ as in Theorem 6.2 below has also been obtained independently in [5]. However, 
[5] focuses just on the case of the logarithm while our work aims at a general approach to the domain descriptions by means of suitable functional calculi.

Note that the results of [11] and [5] hold for Banach spaces over either the real or the complex numbers, while we work exclusively with complex Banach spaces, as a matter of convenience.

Notation. For a closed linear operator $A$ on a complex Banach space $X$ we denote by $\operatorname{dom}(A), \operatorname{ran}(A), \operatorname{ker}(A), \operatorname{fix}(A)$ and $\sigma(A)$ the domain, the range, the kernel, the set of fixed points and the spectrum of $A$, respectively. The norm-closure of the range is written as $\overline{\operatorname{ran}}(A)$. The space of bounded linear operators on $X$ is denoted by $\mathcal{L}(X)$. By $\mathbb{D}, \mathbb{T}$ and $S_{\varphi}$ we denote, respectively, the open unit disc, the torus and the sector $\{z \in \mathbb{C}:|\arg z|<\varphi\}$ in the complex plane. For a sequence $\left(x_{n}\right)_{n \geq 0} \subset X$ we denote by $\mathrm{C}-\lim _{n \rightarrow \infty} x_{n}$ its Cesàro limit whenever the limit exists. If $\alpha=\left(\alpha_{k}\right)_{k=0}^{\infty}$ is a scalar sequence we write $\widehat{\alpha}(z):=\sum_{k=0}^{\infty} \alpha_{k} z^{k}$ for the associated power series.

2. Functional calculus for power-bounded operators. In this section we set up a functional calculus for power-bounded operators. The most natural approach uses power series representations of functions and is presented first. Afterwards we sketch the connection with the functional calculus for sectorial operators.

For $\alpha \in \ell^{1}$ its associated power series

$$
\widehat{\alpha}(z)=\sum_{k=0}^{\infty} \alpha_{k} z^{k}
$$

represents a function on $\overline{\mathbb{D}}$ and we let

$$
\mathrm{A}_{+}^{1}(\mathbb{D}):=\left\{\widehat{\alpha}: \alpha \in \ell^{1}\right\}
$$

be the totality of all these functions. If $f=\widehat{\alpha} \in \mathrm{A}_{+}^{1}(\mathbb{D})$ then $f$ is holomorphic on $\mathbb{D}$, continuous on $\overline{\mathbb{D}}$, and $\alpha_{k}$ is the $k$ th Taylor coefficient of $f$ :

$$
\alpha_{k}=\widehat{f}(k)=\frac{1}{2 \pi i} \int_{\partial \mathbb{D}} f(z) z^{-(k+1)} d z, \quad k \geq 0 .
$$

On $\mathrm{A}_{+}^{1}(\mathbb{D})$ we introduce the norm

$$
\|f\|_{\mathrm{A}_{+}^{1}}:=\|\alpha\|_{\ell^{1}}=\sum_{k=0}^{\infty}\left|\alpha_{k}\right| \quad \text { if } f=\widehat{\alpha} .
$$

It is well-known that $A_{+}^{1}(\mathbb{D})$ is a unital commutative Banach algebra with respect to usual multiplication, isomorphic to the convolution algebra $\ell^{1}$ by virtue of the product identity

$$
\widehat{\alpha * \beta}(z)=\widehat{\alpha}(z) \widehat{\beta}(z), \quad \alpha, \beta \in \ell^{1}, z \in \mathbb{D} .
$$


Now let $X$ be a Banach space. Recall that an operator $T \in \mathcal{L}(X)$ is said to be power-bounded if

$$
M(T):=\sup _{n \in \mathbb{N}_{0}}\left\|T^{n}\right\|<\infty .
$$

If $T$ is power-bounded and $f=\widehat{\alpha} \in \mathrm{A}_{+}^{1}(\mathbb{D})$ we define

$$
f(T):=\sum_{k=0}^{\infty} \alpha_{k} T^{k}
$$

the sum being convergent in operator norm. The following proposition states that power-bounded operators admit a bounded $\mathrm{A}_{+}^{1}(\mathbb{D})$-calculus. We omit its straightforward proof.

Proposition 2.1. Let $T$ be a power-bounded operator on a Banach space $X$. Then the mapping $f \mapsto f(T): \mathrm{A}_{+}^{1}(\mathbb{D}) \rightarrow \mathcal{L}(X)$ is a homomorphism of Banach algebras such that

$$
\|f(T)\| \leq M(T)\|f\|_{\mathrm{A}_{+}^{1}}, \quad f \in \mathrm{A}_{+}^{1}(\mathbb{D}) .
$$

We call this homomorphism the primary functional calculus for $T$. Let us give an important example.

EXAmple 2.2. For $s \in \mathbb{C}$ we write

$$
f_{s}(z):=(1-z)^{s}=\sum_{n=0}^{\infty} \alpha_{n}^{(s)} z^{n}, \quad z \in \mathbb{D} .
$$

If $0<s<1$ and $n \geq 1$ then by the binomial series

$$
\alpha_{n}^{(s)}=\left(\begin{array}{l}
s \\
n
\end{array}\right)(-1)^{n}=\frac{(-s)(1-s) \cdots(n-1-s)}{n !} \leq 0 .
$$

Since $\alpha_{0}^{(s)}=1$, this implies that $\left\|\alpha^{(s)}\right\|_{\ell^{1}}=2$ and so $f_{s} \in \mathrm{A}_{+}^{1}(\mathbb{D})$. Since $f_{s}^{n}=f_{n s}$ for every $n \in \mathbb{N}$ we obtain $f_{s} \in \mathrm{A}_{+}^{1}(\mathbb{D})$ for every $s>0$. We shall see below that the same is true even for $s$ with $\operatorname{Re} s>0$.

One may view Proposition 2.1 as a transference principle. Namely, consider as a special case of the above situation the left shift operator $\tau$ on $X_{0}:=\ell^{1}(\mathbb{Z})$ given by $\tau e_{j}=e_{j-1}, j \in \mathbb{Z}$. Then

$$
\widehat{\alpha}(\tau) \beta=\alpha * \beta, \quad \alpha \in \ell^{1}, \beta \in X_{0},
$$

i.e., $\widehat{\alpha}(\tau)$ is just convolution with $\alpha$. This implies that

$$
\|f\|_{\mathrm{A}_{+}^{1}(\mathbb{D})}=\|f(\tau)\|_{\mathcal{L}\left(X_{0}\right)}
$$

that is, for the special case $T=\tau$ the functional calculus above is actually an isometric embedding. Proposition 2.1 can then be rephrased as

$$
\|f(T)\|_{\mathcal{L}(X)} \leq M(T)\|f(\tau)\|_{\mathcal{L}\left(X_{0}\right)}, \quad f \in \mathrm{A}_{+}^{1}(\mathbb{D}) .
$$


This means that the special case $T=\tau$ has all features (in terms of operator norms) of the general case and it depends much on the context or on personal taste whether to prove a general result directly or just for the operator $\tau$.

The next simple lemma is a consequence of the mean ergodic theorem (see e.g. [23]).

Lemma 2.3. Let $T$ be a power-bounded operator on a Banach space $X$. Then

(a) $\sigma_{p}(T) \cap \mathbb{T} \subset \sigma_{p}\left(T^{*}\right) \cap \mathbb{T}$.

(b) If $X$ is reflexive, then $\sigma_{p}(T) \cap \mathbb{T}=\sigma_{p}\left(T^{*}\right) \cap \mathbb{T}$.

By the lemma, if $I-T$ has dense range, then it is also injective, and if $X$ is reflexive also the converse is true.

In the following we shall always suppose that the operator $A:=I-T$ has a trivial kernel, i.e. $\operatorname{fix}(T)=\{0\}$. Under this hypothesis we are going to extend the primary functional calculus defined in Proposition 2.1 to unbounded functions. The way to do this is canonical and described in depth in [13, Chapters 1 and 2].

Definition 2.4. A function $f$ on $\mathbb{D}$ is called regularizable if there exists $e \in \mathrm{A}_{+}^{1}(\mathbb{D})$ such that $e f \in \mathrm{A}_{+}^{1}(\mathbb{D})$ as well and $e(T)$ is an injective operator. In this case we define

$$
f(T):=e(T)^{-1}(e f)(T)
$$

with its natural domain.

The operator $f(T)$ is closed as the product of a closed and a bounded operator. The definition is independent of the chosen regularizer $e$, as is easily seen [13, Chapter 1]. The class of functions $f$ such that $f(T)$ is defined by this method forms an algebra, and there are specific rules governing this extended functional calculus (see [13, Chapter 1]). Note that whereas the primary functional calculus is the same for all power-bounded operators, the extended functional calculus depends on the particular operator $T$.

Examples 2.5. (1) Consider

$$
f(z)=\log (1-z)=-\sum_{n=1}^{\infty} \frac{1}{n} z^{n}, \quad z \in \mathbb{D} .
$$

Then

$$
(1-z) f(z)=-z\left(1-\sum_{n \geq 1} \frac{1}{n(n+1)} z^{n}\right) \in \mathrm{A}_{+}^{1}(\mathbb{D}) .
$$

Hence $\log (1-z)$ is regularizable by $e(z)=1-z$, since $(1-z)(T)=I-T$ is injective by our standing assumption. 
(2) Using the notation above we have

$$
f_{-s}(z)=(1-z)^{-s}=\sum_{n=0}^{\infty} \alpha_{n}^{(-s)} z^{n}
$$

for $s>0$ with

$$
\alpha_{n}^{(-s)}=\left(\begin{array}{c}
-s \\
n
\end{array}\right)(-1)^{n}=\frac{s(s+1) \cdots(s+n-1)}{n !} \geq 0, \quad n \geq 0 .
$$

If $N>s$ then $(1-z)^{N} f_{-s}=f_{N-s} \in \mathrm{A}_{+}^{1}(\mathbb{D})$, and since $(1-z)^{N}(T)=(I-T)^{N}$ is injective (standing assumption), $f_{-s}(T)$ is defined by regularization. Actually, by standard functional calculus [13, Theorem 1.3.2],

$$
f_{-s}(T)=f_{s}(T)^{-1}, \quad s \in \mathbb{R} .
$$

It seems reasonable to write

$$
\log (I-T)=[\log (1-z)](T), \quad(I-T)^{-s}=\left[(1-z)^{-s}\right](T) .
$$

This was done in [11] and in subsequent papers [1], [6]-[8]. However, logarithms as well as fractional powers are usually defined for so-called sectorial operators in a different way. In the following section we shall see that the two approaches coincide.

3. Sectorial operators. Let us begin with an elementary resolvent estimate.

Lemma 3.1. Let $T$ be a power-bounded operator on a Banach space $X$, and let $A:=I-T$. Then

$$
\|R(\lambda, A)\| \leq \frac{M(T)}{|\lambda-1|-1} \leq \frac{M(T)}{|\operatorname{Re} \lambda|}, \quad \operatorname{Re} \lambda<0 .
$$

Proof. If $|\mu|>1$ then $R(\mu, T)=\sum_{k=0}^{\infty}(1 / \mu)^{k+1} T^{k}$. Hence $\|R(\mu, T)\| \leq$ $M /(|\mu|-1)$. Since $R(\lambda, A)=-R(1-\lambda, T)$, the assertion follows.

A closed operator $A$ on $X$ is said to be sectorial of angle $\omega$ if $\sigma(A) \subset \overline{S_{\omega}}$ and $\sup \left\{\|\lambda R(\lambda, A)\|: \lambda \in \mathbb{C} \backslash \overline{S_{\omega^{\prime}}}\right\}$ is finite for every $\omega^{\prime} \in(\omega, \pi)$. A consequence of Lemma 3.1 is that, for a power-bounded operator $T$, the operator $A=I-T$ is sectorial of angle (at most) $\pi / 2$.

For an (injective) sectorial operator $A$ one has classical definitions of its fractional powers $A^{s}, s \in \mathbb{C}$, and $\log A$ as closed operators [13, Section 3.6]. Equivalently, these operators are obtained by means of a certain "natural" functional calculus for sectorial operators, extensively developed in [13. Chapter 2]. We shall show that the $\mathrm{A}_{+}^{1}(\mathbb{D})$-functional calculus for $T$ is compatible with this "sectorial" functional calculus for $A=I-T$. To this end we briefly recall its construction. 
For $\varphi \in(\omega, \pi)$ denote by $\mathcal{E}_{0}\left(S_{\varphi}\right)$ the set of holomorphic functions on $S_{\varphi}$ such that

$$
|f(z)| \leq C|z|^{s}, \quad z \in S_{\varphi} \cap \mathbb{D},
$$

for some $s>0$ and $C>0$ depending on $f$. Fix some $\omega^{\prime} \in(\omega, \pi)$ and let the contour $\Gamma$ consist of the two segments $\left\{r e^{ \pm i \omega^{\prime}}: r \in\left[0, r_{0}\right]\right\}$ as well as the arc $\left\{r_{0} e^{i r}: r \in\left[-\omega^{\prime}, \omega^{\prime}\right]\right\}$, oriented so that it surrounds the enclosed region once in positive direction. It follows from Cauchy's theorem that

$$
f(z)=\frac{1}{2 \pi i} \int_{\Gamma} \frac{f(\lambda)}{\lambda-z} d \lambda
$$

for every $z$ in the interior of $\Gamma$. If $A$ is sectorial of angle $\omega$ and has spectral radius $r(A)<r_{0}$ then we define

$$
f(A)=\frac{1}{2 \pi i} \int_{\Gamma} f(\lambda) R(\lambda, A) d \lambda .
$$

The sectoriality of $A$ implies that the integral converges in $\mathcal{L}(X)$ and Cauchy's theorem implies that this definition of $f(A)$ is independent of the chosen parameters $\omega^{\prime}$ and $r_{0}$. Standard arguments show that the mapping $f \mapsto f(A), \mathcal{E}_{0}\left(S_{\varphi}\right) \rightarrow \mathcal{L}(X)$ is an algebra homomorphism, the primary functional calculus for the bounded sectorial operator $A$. This functional calculus coincides with the Dunford - Riesz calculus on the common domain. In particular, for a polynomial $p, p(A)$ keeps its usual meaning.

If $\operatorname{ker}(A)=\{0\}$, the primary calculus induces an extended functional calculus by regularization, as above. Basically, one regularizes with powers of $A$ so that every holomorphic function on the sector growing at most polynomially at 0 becomes regularizable. See [13, p. 46] for details.

It has been shown in [13, Proposition 1.2.7] that compatibility carries over from primary to extended functional calculi. So we are reduced to showing the following.

Proposition 3.2. Let $\varphi \in(\pi / 2, \pi), f \in \mathcal{E}_{0}\left(S_{\varphi}\right)$, and $g(z):=f(1-z)$. Then $g \in \mathrm{A}_{+}^{1}(\mathbb{D})$ and

$$
g(T)=f(A)
$$

(the left being defined as in (2.1) and the right being defined by (3.2)), where $T$ is any power-bounded operator on a Banach space $X$ and $A=I-T$.

Proof. The argument is simple and illustrates the power of the above transference principle. Note that for $\Gamma$ in definition (3.2) we have to take $\pi / 2<\omega^{\prime}<\varphi$ and $r_{0}>2$. Then for $\lambda \in \Gamma \backslash\{0\}$ the function

$$
s_{\lambda}(z):=\frac{1}{\lambda-(1-z)}, \quad z \in \mathbb{D},
$$


is in $\mathrm{A}_{+}^{1}(\mathbb{D})$ and $s_{\lambda}(T)=R(\lambda, A)$. Now consider the integral

$$
g(z)=f(1-z)=\frac{1}{2 \pi i} \int_{\Gamma} f(\lambda) s_{\lambda}(z) d \lambda .
$$

This integral converges in $\mathrm{A}_{+}^{1}(\mathbb{D})$ since replacing $z$ by $\tau$ (the left shift on $X_{0}=\ell^{1}(\mathbb{Z})$ ) makes it convergent in $\mathcal{L}\left(X_{0}\right)$, and the $\mathrm{A}_{+}^{1}(\mathbb{D})$-functional calculus for $\tau$ is an isometric embedding. But since the $\mathrm{A}_{+}^{1}(\mathbb{D})$-functional calculus for $T$ is continuous, one obtains

$$
g(T)=\frac{1}{2 \pi i} \int_{\Gamma} f(\lambda) s_{\lambda}(T) d \lambda=\frac{1}{2 \pi i} \int_{\Gamma} f(\lambda) R(\lambda, A) d \lambda=f(A)
$$

as desired.

As an application we note first that if $\operatorname{Re} s>0$ then $z^{s} \in \mathcal{E}_{0}\left(S_{\pi}\right)$ and by Proposition 3.2 we have $f_{s}(z)=(1-z)^{s} \in \mathrm{A}_{+}^{1}(\mathbb{D})$ and $f_{s}(T)=(I-T)^{s}$. Passing to the extended calculi we obtain

$$
[\log (1-z)](T)=\log (I-T) \quad \text { and } \quad\left[(1-z)^{s}\right](T)=(I-T)^{s}, \quad s \in \mathbb{C},
$$

as desired. The family $\left((I-T)^{s}\right)_{\operatorname{Re} s>0}$ is a holomorphic semigroup [13, Prop. 3.1.1], which is strongly continuous as $s \searrow 0$ if and only if $\operatorname{ran}(I-T)$ is dense in $X$ [13, Prop. 3.1.15]. Its generator (defined via the Laplace transform) is $G:=\log (I-T)$ [13, Prop. 3.5.3], a fact that goes back to [26].

4. Admissible functions and log-convex sequences. After these functional calculus preliminaries we approach the main objective of our paper. Let $T$ be a power-bounded operator $T$ on a Banach space $X$ and let $f$ be a holomorphic function on $\mathbb{D}$ with Taylor expansion $f(z)=\sum_{k=0}^{\infty} \alpha_{k} z^{k}$. Then for a vector $x \in X$ we ask whether $x \in \operatorname{dom}(f(T))$ is characterized by the convergence (in one way or another) of the series

$$
\sum_{k=0}^{\infty} \alpha_{k} T^{k} x
$$

Unfortunately, we are not able to answer this question in its full generality. Instead, we restrict ourselves to the case that $f$ is regularizable by $e(z)=$ $1-z$ as in the motivating examples. It turns out that then a weak hypothesis about the convergence of the series already implies that $x \in \operatorname{dom}(f(T))$ (see Proposition 5.1 below). The idea for the converse implication is to write

$$
f_{n}(z):=\sum_{k=0}^{n-1} \alpha_{k} z^{k}=\left[\frac{1}{f(z)} \sum_{k=0}^{n-1} \alpha_{k} z^{k}\right] f(z)=g_{n}(z) f(z)
$$

if $1 / f \in \mathrm{A}_{+}^{1}(\mathbb{D})$. Under certain conditions on the Taylor coefficients of $1 / f$ we shall show that $\sup _{n \geq 1}\left\|g_{n}\right\|_{\mathrm{A}_{+}^{1}}<\infty$ and that $\left(g_{n}\right)_{n \geq 1}$ forms an "approx- 
imation of the identity" in $\mathrm{A}_{+}^{1}(\mathbb{D})$, in some sense. Transferring this via the functional calculus yields the desired statement for $f(T)$.

Definition 4.1. Let $f(z):=\sum_{k=0}^{\infty} \alpha_{k} z^{k}$ be a holomorphic function on $\mathbb{D}$. The function $f$ (or, equivalently, the sequence $\left.\left(\alpha_{k}\right)_{k \geq 0}\right)$ is called $a d$ missible if $\alpha_{k} \geq 0$ for all $k \geq 0, f$ does not have zeros in $\mathbb{D}$, and

$$
\frac{-1}{f(z)}=\sum_{k=0}^{\infty} \beta_{k} z^{k}, \quad z \in \mathbb{D},
$$

with $\beta_{k} \geq 0$ for all $k \geq 1$.

EXAMPLE 4.2. If $f(z)=f_{-s}(z)=(1-z)^{-s}$ for $0<s<1$, then $f$ is admissible because

$$
\frac{1}{f_{-s}(z)}=f_{s}(z)=\sum_{k=0}^{\infty} \alpha_{k}^{(s)} z^{k}=1-\sum\left(-\alpha_{k}^{(s)}\right) z^{k}
$$

and $-\alpha_{k}^{(s)} \geq 0$ (cf. Example 2.5(2)).

In the following we describe a large class of admissible functions. A sequence $\alpha=\left(\alpha_{k}\right)_{k \geq 0}$ is called logarithmically convex or just log-convex if $\alpha_{k} \geq 0$ for all $k \geq 0$ and

$$
\alpha_{k}^{2} \leq \alpha_{k-1} \alpha_{k+1}, \quad k \geq 1 .
$$

Log-convex sequences are sometimes called Kaluza sequences. They form a convex cone, a fact which is seen by employing the 2-dimensional CauchySchwarz inequality. A log-convex sequence $\left(\alpha_{k}\right)_{k \geq 0}$ is either identically zero or $\alpha_{k}>0$ for all $k \in \mathbb{N}_{0}$. In the latter case, log-convexity is obviously equivalent to the sequence $\left(\alpha_{n+1} / \alpha_{n}\right)_{n \geq 0}$ being monotonically increasing.

By direct computation one checks that for $s>0$ the sequences

$$
\left((n+1)^{-s}\right)_{n \geq 0} \quad \text { and } \quad \alpha_{n}^{(-s)}=\frac{s(s+1) \ldots(s+n-1)}{n !}, \quad n \geq 0,
$$

are log-convex. If $\alpha$ is log-convex then $\left(\alpha_{n}^{s}\right)_{n}$ is log-convex for every $s>0$. A typical example of a log-convex sequence is the sequence of moments

$$
\alpha_{k}:=\int_{0}^{1} t^{k} \mu(d t), \quad k \geq 0,
$$

of a positive finite Borel measure $\mu$ on $[0,1]$. (This, again, follows from the Cauchy-Schwarz inequality.) For more details on the structure of log-convex sequences see e.g. [21] and [22].

Suppose that $\alpha$ is log-convex and define

$$
f(z):=\widehat{\alpha}(z)=\sum_{k=0}^{\infty} \alpha_{k} z^{k}, \quad z \in \mathbb{D} .
$$


Then we have $f=0$ or $\alpha_{k}>0$ for all $k \in \mathbb{N}_{0}$. As the quotients $\alpha_{k+1} / \alpha_{k}$ are increasing, they converge to a limit $l$, which by elementary complex analysis is the inverse of the radius of convergence of the power series defining $f$. As we are mainly interested in functions being defined on $\mathbb{D}$, we shall consider only the case $l \leq 1$, i.e., $\left(\alpha_{k}\right)_{k \geq 0}$ is decreasing. In this case, $f$ is regularizable by $e(z)=1-z$, as the following simple lemma shows.

Lemma 4.3. Suppose that $f(z)=\sum_{k=0}^{\infty} \alpha_{k} z^{k}$ such that $0 \leq \alpha_{k+1} \leq \alpha_{k}$. Then $f$ has an extension to a continuous function on $\overline{\mathbb{D}} \backslash\{1\}$, and $(1-z)$. $f(z) \in \mathrm{A}_{+}^{1}(\mathbb{D})$. Furthermore, $0 \leq f(t)$ is increasing in $t \in[0,1]$ and either $f \in \mathrm{A}_{+}^{1}(\mathbb{D})$ with $\|f\|_{\mathrm{A}_{+}^{1}}=f(1):=\lim _{t / 1} f(t)$, or $f(1):=\lim _{t \nearrow 1} f(t)=+\infty$.

Proof. Clearly $(1-z) f(z)=\alpha_{0}+\sum_{k \geq 1}\left(\alpha_{k}-\alpha_{k-1}\right) z^{k}$. Since $\alpha$ is decreasing, $\sum_{k}\left|\alpha_{k}-\alpha_{k-1}\right|<\infty$. The rest is clear.

The following result states that decreasing log-convex sequences are admissible. It is originally due to Kaluza [20] (see also [29] and [15, Chapter IV, Theorem 22]). For the convenience of the reader we include the short proof.

Proposition 4.4 (Kaluza). Let $\alpha=\left(\alpha_{k}\right)_{k \geq 0}$ be a decreasing log-convex sequence and let $f(z):=\sum_{k=0}^{\infty} \alpha_{k} z^{k}, z \in \mathbb{D}$. Then $f$ and $\alpha$ are admissible. More precisely, $f$ does not have a zero in $\mathbb{D}$ and

$$
\frac{1}{f(z)}=-\sum_{k=0}^{\infty} \beta_{k} z^{k}, \quad z \in \mathbb{D}
$$

with $\beta_{0}=-1 / \alpha_{0} \leq 0$ and $0 \leq \beta_{k} \leq \alpha_{k} / \alpha_{0}^{2}$ for all $k \geq 1$.

Proof. Since $f(0)=\alpha_{0}>0,-1 / f(z)$ has a power series representation

$$
-1 / f(z)=\sum_{k \geq 0} \beta_{k} z^{k}
$$

around zero. Clearly $\beta_{0}=-1 / \alpha_{0}, \beta_{1}=\alpha_{1} / \alpha_{0}^{2}$ and

$$
0=\alpha_{n} \beta_{0}+\sum_{j=1}^{n} \alpha_{n-j} \beta_{j}, \quad 0=\alpha_{n+1} \beta_{0}+\sum_{j=1}^{n+1} \alpha_{n+1-j} \beta_{j}
$$

for all $n \geq 1$. Multiplying the first identity by $\alpha_{n+1}$ and the second by $\alpha_{n}$ and subtracting yields

$$
\begin{aligned}
0 & =\sum_{j=1}^{n} \alpha_{n-j} \alpha_{n+1} \beta_{j}-\sum_{j=1}^{n+1} \alpha_{n+1-j} \alpha_{n} \beta_{j} \\
& =-\alpha_{0} \alpha_{n} \beta_{n+1}+\sum_{j=1}^{n}\left(\alpha_{n-j} \alpha_{n+1}-\alpha_{n+1-j} \alpha_{n}\right) \beta_{j},
\end{aligned}
$$


which leads to

$$
\beta_{n+1}=\frac{1}{\alpha_{0}} \sum_{j=1}^{n}\left(\frac{\alpha_{n+1}}{\alpha_{n}}-\frac{\alpha_{n-j+1}}{\alpha_{n-j}}\right) \alpha_{n-j} \beta_{j} \geq 0
$$

by the log-convexity of $\alpha$. Hence by induction $\beta_{k} \geq 0$ for all $k \geq 1$. Moreover, using this we obtain

$$
\beta_{n}=\frac{-1}{\alpha_{0}}\left(\alpha_{n} \beta_{0}+\sum_{j=1}^{n-1} \alpha_{j} \beta_{n-j}\right)=\frac{\alpha_{n}}{\alpha_{0}^{2}}-\frac{1}{\alpha_{0}} \sum_{j=1}^{n-1} \alpha_{j} \beta_{n-j} \leq \frac{\alpha_{n}}{\alpha_{0}^{2}}
$$

for all $n \geq 1$. Therefore, the radius of convergence of the power series for $1 / f$ is greater than or equal to 1 , and therefore $f$ has no zeros in $\mathbb{D}$.

By virtue of Kaluza's theorem, admissible sequences abound. We hence return to the general situation.

Lemma 4.5. Let $f$ be admissible. Then $1 / f \in \mathrm{A}_{+}^{1}(\mathbb{D})$ and

$$
\|1 / f\|_{\mathrm{A}_{+}^{1}}=\frac{2}{f(0)}-\frac{1}{f(1)}
$$

where $1 / \infty:=0$. Moreover, $f$ does not have a zero on $\overline{\mathbb{D}}$. Then

Proof. Write as above $-1 / f(z)=\sum_{k=0}^{\infty} \beta_{k} z^{k}=-1 / f(0)+\sum_{k \geq 1} \beta_{k} z^{k}$.

$$
\sum_{k \geq 1} \beta_{k}=\lim _{t \nearrow 1} \sum_{k \geq 1} \beta_{k} t^{k}=\frac{1}{\alpha_{0}}-\lim _{t \nearrow 1} \frac{1}{f(t)}=\frac{1}{f(0)}-\frac{1}{f(1)}<\infty .
$$

Hence $\beta \in \ell^{1}$ and

$$
\|1 / f\|_{\mathrm{A}_{+}^{1}}=\|\beta\|_{1}=\left|\beta_{1}\right|+\sum_{k \geq 1} \beta_{k}=\frac{1}{f(0)}+\frac{1}{f(0)}-\frac{1}{f(1)}=\frac{2}{f(0)}-\frac{1}{f(1)}
$$

as claimed. This shows that $1 / f$ extends continuously to $\overline{\mathbb{D}}$, whence $f$ cannot have a zero even on $\partial \mathbb{D}=\mathbb{T}$.

For an admissible $\alpha$ consider

$$
g_{n}(z):=\frac{1}{f(z)} \sum_{k=0}^{n-1} \alpha_{k} z^{k}, \quad n \geq 1, z \in \mathbb{D} .
$$

Clearly $g_{n} \rightarrow \mathbf{1}$ pointwise on $\mathbb{D}$.

Lemma 4.6. For every $n \in \mathbb{N}$,

$$
g_{n}(z)=1-\sum_{k=n}^{\infty} \gamma_{k, n} z^{k} \quad \text { with } \quad \gamma_{k, n} \geq 0
$$


Furthermore,

$$
\left\|g_{n}-1\right\|_{\mathrm{A}_{+}^{1}}=1-g_{n}(1)=1-\frac{1}{f(1)} \sum_{k=0}^{n-1} \alpha_{k}
$$

and

$$
\left\|g_{n}\right\|_{\mathrm{A}_{+}^{1}}=2-g_{n}(1)=2-\frac{1}{f(1)} \sum_{k=0}^{n-1} \alpha_{k} .
$$

Proof. On the one hand,

$$
g_{n}(z)=\frac{1}{f(z)} \sum_{k=0}^{n-1} \alpha_{k} z^{k}=\frac{1}{f(z)}\left(f(z)-\sum_{k=n}^{\infty} \alpha_{k} z^{k}\right)=1-\frac{1}{f(z)} \sum_{k=n}^{\infty} \alpha_{k} z^{k},
$$

and on the other hand,

$$
g_{n}(z)=-\left(\sum_{j=0}^{\infty} \beta_{j} z^{j}\right)\left(\sum_{k=0}^{n-1} \alpha_{k} z^{k}\right)=1-\sum_{k=1}^{n-1} \beta_{0} \alpha_{k} z^{k}-\sum_{j \geq 1,0 \leq k<n} \beta_{j} \alpha_{k} z^{k+j} .
$$

The first representation tells us that the $k$ th Taylor coefficient of $g_{n}$ vanishes for $k=1, \ldots, n-1$. Hence

$$
g_{n}(z)=1-\sum_{k=n}^{\infty} \gamma_{k, n} z^{k}
$$

and, from the second representation,

$$
\gamma_{k, n}=\sum_{j=0}^{n-1} \alpha_{j} \beta_{k-j} \geq 0, \quad k \geq n,
$$

since $\beta_{j} \geq 0$ for all $j \geq 1$ and $\alpha_{k} \geq 0$ for all $k \geq 0$. This implies

$$
\left\|g_{n}-1\right\|_{\mathrm{A}_{+}^{1}}=\sum_{k \geq n} \gamma_{k, n}=1-g_{n}(1)
$$

and

$$
\left\|g_{n}\right\|_{\mathrm{A}_{+}^{1}}=1+\sum_{k \geq n} \gamma_{k, n}=2-g_{n}(1) .
$$

Now we are ready to prove our main approximation result.

Corollary 4.7. Suppose that $f$ is an admissible function with Taylor coefficients $\alpha$, and let $g_{n}$ be defined as above. Then the following assertions hold.

(a) If $f(1)<\infty$ then $g_{n} \rightarrow \mathbf{1}$ in $\mathrm{A}_{+}^{1}(\mathbb{D})$ as $n \rightarrow \infty$.

(b) If $f(1)=\infty$ then $\left\|g_{n}\right\|_{\mathrm{A}_{+}^{1}}=2$ for all $n$.

(c) If $(1-z) f(z) \in \mathrm{A}_{+}^{1}(\mathbb{D})$ then

$$
\underset{n \rightarrow \infty}{\mathrm{C}-\lim _{n}}(1-z) g_{n}(z)=1-z \quad \text { in } \mathrm{A}_{+}^{1}(\mathbb{D}) .
$$


(d) If $(1-z) f(z) \in \mathrm{A}_{+}^{1}(\mathbb{D})$ and $\lim _{n \rightarrow \infty} \alpha_{n}=0$ then

$$
\lim _{n \rightarrow \infty}(1-z) g_{n}(z)=1-z \quad \text { in } \mathrm{A}_{+}^{1}(\mathbb{D}) .
$$

Proof. If $f(1)<\infty$ then $\|\alpha\|_{1}=f(1)<\infty$ and

$$
g_{n}(1)-1=f(1)^{-1}\left(\sum_{k=n+1}^{\infty} \alpha_{k}\right) \rightarrow 0, \quad n \rightarrow \infty .
$$

Lemma 4.6 implies the statement in (a). In order to prove (b), suppose that $f(1)=\infty$. Then Lemma 4.6 shows that $\left\|g_{n}\right\|_{\mathrm{A}_{+}^{1}}=2$ for all $n \geq 1$. Write

$$
\begin{aligned}
(1-z) g_{n}(z) & =\frac{1}{f(z)}\left(\alpha_{0}+\sum_{k=1}^{n-1}\left(\alpha_{k}-\alpha_{k-1}\right) z^{k}\right)-z \frac{\alpha_{n-1} z^{n-1}}{f(z)} \\
& =h_{n}(z)+r_{n}(z) .
\end{aligned}
$$

Since $(1-z) f \in \mathrm{A}_{+}^{1}(\mathbb{D})$, we have $h_{n} \rightarrow(1-z) f / f=1-z$ in $\mathrm{A}_{+}^{1}(\mathbb{D})$ as $n \rightarrow \infty$. For the Cesàro means of $\left(r_{n}\right)_{n \geq 1}$ we obtain

$$
\frac{1}{N} \sum_{n=1}^{N} r_{n}(z)=z \frac{g_{N}(z)}{N} \rightarrow 0, \quad N \rightarrow \infty,
$$

in $\mathrm{A}_{+}^{1}(\mathbb{D})$, since $\left\|g_{N}\right\|_{\mathrm{A}_{+}^{1}}=2$ for all $N \geq 1$. If $\lim _{n \rightarrow \infty} \alpha_{n}=0$, then $r_{n}$ obviously tends to 0 in $\|\cdot\|_{A_{+}^{1}(\mathbb{D})}$.

5. Partial sum approximations for power-bounded operators. Let us now return to our main subject. First we will characterize the domain of $f(T)$ by means of the extrapolation norm $\|x\|_{-1}:=\|(I-T) x\|, x \in X$, weaker than the original norm on $X$. This result nevertheless will be useful for obtaining a characterization of the domain in the original norm as well.

Proposition 5.1. Let $f: \mathbb{D} \rightarrow \mathbb{C}$ be holomorphic such that $(1-z) f(z) \in$ $\mathrm{A}_{+}^{1}(\mathbb{D})$, and let $T$ be a power-bounded operator on a Banach space $X$ with $\operatorname{fix}(T)=0$.

(a) If $x \in X$ is such that there is $y \in X$ with

$$
\underset{n \rightarrow \infty}{\mathrm{C}-\lim _{m}}(I-T) \sum_{k=0}^{n} \alpha_{k} T^{k} x=(I-T) y \quad \text { weakly, }
$$

then $x \in \operatorname{dom}(f(T))$ and $f(T) x=y$.

(b) If $x \in \operatorname{dom}(f(T))$ is such that $\mathrm{C}-\lim _{n \rightarrow \infty} \alpha_{n} T^{n+1} x=0$ weakly, then

$$
\underset{n \rightarrow \infty}{\mathrm{C}-\lim _{n}}(I-T) \sum_{k=0}^{n} \alpha_{k} T^{k} x=(I-T) f(T) x \quad \text { weakly. }
$$


The result of (b) is also true on replacing weak convergence by strong convergence, and/or Cesàro convergence by ordinary convergence.

Proof. To prove (a), note that (5.1) implies that C- $\lim _{n \rightarrow \infty}(I-T) \alpha_{n} T^{n} x$ $=0$ weakly. Next, since

$$
(1-z) \sum_{k=0}^{n} \alpha_{k} z^{k}=\alpha_{0}+\sum_{k=1}^{n}\left(\alpha_{k}-\alpha_{k-1}\right) z^{k}-\alpha_{n} z^{n+1},
$$

we have

$$
(1-z) f(z)=\alpha_{0}+\sum_{k=1}^{\infty}\left(\alpha_{k}-\alpha_{k-1}\right) z^{k}, \quad z \in \mathbb{D},
$$

with $\sum_{k \geq 1}\left|\alpha_{k}-\alpha_{k-1}\right|<\infty$ by hypothesis. Hence

$$
\lim _{n \rightarrow \infty}\left(\alpha_{0}+\sum_{k=1}^{n}\left(\alpha_{k}-\alpha_{k-1}\right) T^{k}\right)=[(1-z) f](T)
$$

in operator norm. This implies that $\mathrm{C}-\lim _{n \rightarrow \infty} \alpha_{n} T^{n+1} x=z$ weakly for some $z \in X$. But then

$$
\begin{aligned}
(I-T) z & =\underset{n \rightarrow \infty}{\mathrm{C}-\lim _{n}}(I-T) \alpha_{n} T^{n+1} x \\
& =T \underset{n \rightarrow \infty}{\mathrm{C}-\lim _{n}(I-T) \alpha_{n} T^{n} x=0 \quad \text { weakly }}
\end{aligned}
$$

which yields $z=0$ since $\operatorname{fix}(T)=\{0\}$. Therefore we obtain

$$
[(1-z) f](T) x=(I-T) y
$$

and this is - by definition via regularization - equivalent to $f(T) x=y$.

Assertion (b) is a consequence of (5.2) and (5.3) and the fact that one has $[(1-z) f](T) x=(I-T) f(T) x$ for $x \in \operatorname{dom}(f(T))$.

If $\lim _{n \rightarrow \infty} \alpha_{n}=0$ we obtain a neat characterization of $\operatorname{dom}(f(T))$.

Corollary 5.2. Let $f, \alpha$ be as in Proposition 5.1 and such that $\lim _{n \rightarrow \infty} \alpha_{n}=0$. Then the condition $x \in \operatorname{dom}(f(T))$ is equivalent to the convergence in $X$ of the series $\sum_{k \geq 1} \alpha_{k} T^{k} x$ with respect to the extrapolation norm $\|\cdot\|_{-1}$.

The next example shows that the condition $x \in \operatorname{dom}(f(T))$ in general is too weak to imply the convergence of the series $\sum_{k=0}^{\infty} \alpha_{k} T^{k} x$.

Example 5.3. Consider $f(z)=(1-z)^{-1}=\sum_{k \geq 0} z^{k}$. Let $x=(I-T) y$. Then the convergence

$$
(I-T) \sum_{k=0}^{n} \alpha_{k} T^{k} x \rightarrow(I-T) y, \quad n \rightarrow \infty,
$$

is equivalent to $T^{n+1} x \rightarrow 0$. But if for example $T=-I$, then $T^{n} x \rightarrow 0$ if and only if $x=0$. 
The following is an operator counterpart of Corollary 4.7, yielding an approximation $\left(g_{n}(T)\right)_{n \geq 1}$ of $I$ with uniformly bounded norms.

Proposition 5.4. Let $f(z)=\sum_{k>0} \alpha_{k} z^{k}$ be admissible, with $(1-z) f \in$ $\mathrm{A}_{+}^{1}(\mathbb{D})$. Let $\left(g_{n}\right)_{n \geq 1} \subset \mathrm{A}_{+}^{1}(\mathbb{D})$ be defined as in 4.1. Suppose that $T$ is a power-bounded operator on $X$ with $\operatorname{fix}(T)=\{0\}$. Then the following assertions hold:

(a) If $f(1)<\infty$ then

$$
\lim _{n \rightarrow \infty} g_{n}(T)=I \quad \text { in operator norm. }
$$

(b) If $f(1)=\infty$ then $\left\|g_{n}(T)\right\| \leq 2 M(T)$ for all $n \geq 1$ and

$$
\mathrm{C}_{n \rightarrow \infty}(I-T) g_{n}(T)=I-T \quad \text { in operator norm. }
$$

(c) If $f(1)=\infty$ and $\lim _{k \rightarrow \infty} \alpha_{k}=0$ then

$$
\lim _{n \rightarrow \infty}(I-T) g_{n}(T)=I-T \quad \text { in operator norm. }
$$

(d) If $f(1)=\infty$ and $x \in X$ is such that $\lim _{n \rightarrow \infty} \alpha_{n} T^{n+1} f(T)^{-1} x=0$ then

$$
\lim _{n \rightarrow \infty}(I-T) g_{n}(T) x=(I-T) x \quad \text { in norm. }
$$

Proof. In view of Proposition 2.1, assertions (a), (b) and (c) follow from (a), (b), and (c) of Corollary 4.7, respectively. To prove (d) we use (4.2) and write

$$
(I-T) g_{n}(T)=h_{n}(T)(I-T)-\alpha_{n-1} T^{n} f(T)^{-1}
$$

where $h_{n}(T) \rightarrow I$ in operator norm. Then the statement follows.

Corollary 5.5. Let $\alpha, f, g_{n}$ and $T, X$ be as in Proposition 5.4. Then

$$
\underset{n \rightarrow \infty}{\mathrm{C}-\lim _{n}} g_{n}(T) x=x, \quad x \in \overline{\operatorname{ran}}(I-T) .
$$

Furthermore, one has

$$
\lim _{n \rightarrow \infty} g_{n}(T) x=x, \quad x \in \overline{\operatorname{ran}}(I-T),
$$

if and only if

$$
\lim _{n \rightarrow \infty} \alpha_{n} T^{n+1} x=0, \quad x \in \overline{\operatorname{ran}}(I-T) .
$$

Proof. The first statement is a direct consequence of Proposition 5.4, as also is the "if" part of the second statement. To prove the "only if" part suppose that $g_{n}(T) x \rightarrow x$ for all $x \in \operatorname{ran}(I-T)$. Then $(I-T) g_{n}(T) \rightarrow$ $(I-T) x$ strongly. Using (5.4) we infer that $\alpha_{n} T^{n+1} f(T)^{-1} \rightarrow 0$ strongly. But since $f(T)(I-T) \in \mathcal{L}(X)$, we have $\operatorname{ran}(I-T) \subset \operatorname{dom}(f(T))$, and hence $\alpha_{n} T^{n+1} \rightarrow 0$ strongly on $\overline{\operatorname{ran}}(I-T)$.

Let us now focus on the most interesting case $f(1)=\infty$. To simplify the formulation of our main result we will state it only for a power-bounded 
operator $T$ such that $\overline{\operatorname{ran}}(I-T)=X$ (so that fix $(T)=\{0\}$ ) and for $\alpha$ tending to zero. This will suffice for the domain descriptions of functions of $T$ given in the next section.

THEOREM 5.6. Let $\left(\alpha_{k}\right)_{k \geq 0}$ be an admissible sequence tending to zero, let $f(z):=\sum_{k \geq 0} \alpha_{k} z^{k}, z \in \mathbb{D}$, and suppose that $f(1)=\infty$ and $(1-z) f \in$ $\mathrm{A}_{+}^{1}(\mathbb{D})$. Let $T$ be a power-bounded operator on a Banach space $X$ such that $\overline{\operatorname{ran}}(I-T)=X$. For $x \in X$ the following assertions are equivalent.

(i) $x \in \operatorname{dom}(f(T))$.

(ii) The series $\sum_{k \geq 0} \alpha_{k} T^{k} x$ Cesàro-converges in norm.

(iii) The series $\sum_{k \geq 0} \alpha_{k} T^{k} x$ converges in norm.

(iv) The series $\sum_{k \geq 0} \alpha_{k} T^{k} x$ Cesàro-converges weakly.

(v) The series $\sum_{k \geq 0} \alpha_{k} T^{k} x$ converges weakly.

If $X$ is reflexive then assertions $(\mathrm{i})-(\mathrm{v})$ are equivalent to

(vi) $\sup _{N}\left\|\sum_{k=0}^{N} \alpha_{k} T^{k} x\right\|<\infty$.

Furthermore, if (i)-(v) hold true, then $f(T) x=\sum_{k=0}^{\infty} \alpha_{k} T^{k} x$.

Proof. To prove the equivalence of (i)-(v) it is enough to show that (i) $\Rightarrow$ (iii) and (iv) $\Rightarrow$ (i).

Let $x \in \operatorname{dom}(f(T))$. By Corollary 5.5 and the hypothesis $\overline{\operatorname{ran}}(I-T)=X$ we see that $g_{n}(T) \rightarrow I$ strongly. General functional calculus theory 13 , Proposition 1.1.2] implies that $g_{n}(T) f(T) \subset\left(g_{n} f\right)(T)$ and hence we obtain

$$
\sum_{k=0}^{n-1} \alpha_{k} T^{k} x=\left(g_{n} f\right)(T) x=g_{n}(T) f(T) x \rightarrow f(T) x, \quad n \rightarrow \infty .
$$

Suppose now that (iv) holds. Then (5.1) holds as well, and Proposition 5.1(a) implies that $x \in \operatorname{dom}(f(T))$.

If $X$ is reflexive and (vi) is true, then the mean ergodicity of $T^{\prime}$ yields $X^{\prime \prime}=\operatorname{fix}\left(T^{\prime}\right) \oplus \overline{\operatorname{ran}}\left(I-T^{\prime}\right)$, and

$$
\operatorname{fix}\left(T^{\prime}\right)=\operatorname{ker}\left(I-T^{\prime}\right)=\operatorname{ran}(I-T)^{\perp}=X^{\perp}=0,
$$

whence $X^{\prime \prime}=\overline{\operatorname{ran}}\left(I-T^{\prime}\right)$. For $x^{\prime \prime}=\left(I-T^{\prime}\right) y^{\prime} \in X^{\prime}$ we have

$$
\left\langle\sum_{k=0}^{n} \alpha_{k} T^{k} x, x^{\prime}\right\rangle=\left\langle\alpha_{0} x+\sum_{k=1}^{n}\left(\alpha_{k}-\alpha_{k-1}\right) T^{k} x, y^{\prime}\right\rangle-\left\langle\alpha_{n} T^{n+1} x, y^{\prime}\right\rangle
$$

where the first summand converges to $\left\langle[(1-z) f](T) x, y^{\prime}\right\rangle$ and the second converges to 0 in the Cesàro sense (using (vi)). Using (vi) again we conclude that the $\operatorname{limit}_{\lim _{n \rightarrow \infty}}\left\langle\sum_{k=0}^{n} \alpha_{k} T^{k} x, x^{\prime}\right\rangle$ exists in the Cesàro sense for all $x^{\prime} \in X^{\prime}$. Hence $\sum_{k=0}^{\infty} \alpha_{k} T^{k} x$ Cesàro converges in the weak* topology of $X^{\prime \prime}$, but as $X$ is reflexive we arrive at (iv). 
Remark 5.7. Let $f: \mathbb{D} \rightarrow \mathbb{C}$ be such that $(1-z) f \in \mathrm{A}_{+}^{1}(\mathbb{D})$. Then $f(T)(I-T)=[(1-z) f](T) \in \mathcal{L}(X)$ and this yields $\operatorname{ran}(I-T) \subset \operatorname{dom}(f(T))$. Hence if $1 \notin \sigma(T)$ then $f(T)$ is a bounded operator.

The converse holds if in addition $f=\widehat{\alpha}$ with $\alpha$ being an admissible null-sequence and $f(1)=\infty$. Indeed, in this case, $1 \in \sigma(T)$ implies that

$$
\left\|\sum_{k=0}^{n} \alpha_{k} T^{k}\right\| \geq \sup _{\lambda \in \sigma(T)}\left|\sum_{k=0}^{n} \alpha_{k} \lambda^{k}\right| \geq \sum_{k=0}^{n} \alpha_{k} \rightarrow \infty
$$

as $n \rightarrow \infty$. This shows that the series $\sum_{k=0}^{\infty} \alpha_{k} T^{k} x$ cannot converge for every $x \in X$. Hence $\operatorname{dom}(f(T)) \neq X$, by Theorem 5.6 .

6. Examples: fractional powers and the logarithm. As the first application of our Corollary 5.6 we characterize the domains of $(I-T)^{-s}$, $s \in(0,1)$, for a power-bounded operator $T$ on $X$ with $\overline{\operatorname{ran}}(I-T)=X$. Since $\operatorname{ran}\left((I-T)^{s}\right)=\operatorname{dom}\left((I-T)^{-s}\right)$ this characterization allows one to obtain a solution $x$ of the equation $(I-T)^{s} x=y$ as a limit of certain averages of $\left\{T^{n} y: n \geq 0\right\}$. These solutions are called $s$-fractional coboundaries for $T$ and are of importance in operator ergodic theory.

Recall from Example 2.5(2) our notation

$$
(1-z)^{-s}=\sum_{k=0}^{\infty} \alpha_{k}^{(-s)} z^{k}, \quad z \in \mathbb{D},
$$

for $s \in \mathbb{R}$. If $s \in(0,1)$ then the sequence $\left(\alpha_{k}^{(-s)}\right)_{k \geq 0}$ is log-convex and decreasing to zero with rate $k^{s-1}$. It follows from a classical asymptotic analysis using [31, part I, p. 77] that

$$
\alpha_{k}^{(-s)}=\frac{1}{\Gamma(s) k^{1-s}}+O\left(k^{s-2}\right)+O\left(k^{-s-1}\right)
$$

(cf. also [11, proof of Theorem 2.11]. The following result was proved in [11] by a subtle analysis of the series expansions for $(1-z)^{s}$ (see [11, Theorems 2.11, 2.13]). Here it becomes a straightforward consequence of our Corollary 5.6 .

TheOREm 6.1. Let $T$ be a power-bounded operator on a Banach space $X$ such that $\overline{\operatorname{ran}}(I-T)=X$, and let $s \in(0,1)$. Then for $x \in X$ the following statements are equivalent.

(i) $x \in \operatorname{ran}(I-T)^{s}$.

(ii) $\sum_{k=1}^{\infty} T^{k} x / k^{1-s}$ converges.

(iii) $\sum_{k=0}^{\infty} \alpha_{k}^{(-s)} T^{k} x$ converges weakly.

(iv) $\sum_{k=0}^{\infty} \alpha_{k}^{(-s)} T^{k} x$ converges. 
If either of (i)-(iv) holds, then $(I-T)^{-s} x=\sum_{k=0}^{\infty} a_{k}^{(-s)} T^{k} x$. If $X$ is reflexive then each of the statements (i)-(iv) is equivalent to

(v) $\sup _{n}\left\|\sum_{k=1}^{n} T^{k} x / k^{1-s}\right\|<\infty$.

Proof. By the above remarks, the equivalence of (i), (iii) and (iv) follows immediately from Theorem 5.6. The equivalence of (ii) and (iv) follows from (6.1), as does the fact that (v) is equivalent to

$$
\sup _{n}\left\|\sum_{k=0}^{n} \alpha_{k}^{(-s)} T^{k} x\right\|<\infty .
$$

But this is equivalent to (i) again by Theorem 5.6 .

Let us turn to the logarithm. The function $\log (1-z)=-\sum_{k=1}^{\infty} z^{k} / k$ is not admissible; however, the function

$$
f(z):=\frac{-\log (1-z)}{z}=\sum_{k=0}^{\infty} \frac{1}{k+1} z^{k}
$$

is, since its Taylor coefficients form a decreasing, log-convex null-sequence. Moreover,

$$
h(z):=\log (1-z)-f(z)=1-\sum_{k=1}^{\infty} \frac{z^{k}}{k(k+1)} \in \mathrm{A}_{+}^{1}(\mathbb{D}) .
$$

Using these preparations, we are now able to identify the operator $\log (I-T)$ as the "one-sided ergodic Hilbert transform"

$$
H_{T}=\sum_{k=1}^{\infty} \frac{1}{k} T^{k}
$$

with its natural domain. As explained in the Introduction, this answers an open question from [11].

THEOREM 6.2. Let $T$ be a power-bounded operator on a Banach space $X$ such that $\overline{\operatorname{ran}}(I-T)=X$. Then $\log (I-T)=H_{T}$. More precisely, for $x \in X$ the following statements are equivalent.

(i) $x \in \operatorname{dom}(\log (I-T))$.

(ii) The series $\sum_{k=1}^{\infty} T^{k} x / k$ converges.

(iii) The series $\sum_{k=1}^{\infty} T^{k} x / k$ converges weakly.

If (i)-(iii) hold, then $\log (I-T) x=-\sum_{k=1}^{\infty} T^{k} x / k$. If $X$ is reflexive, each of the statements (i)-(iii) is equivalent to

(iv) $\sup _{n}\left\|\sum_{k=1}^{n} T^{k} x / k\right\|<\infty$.

Proof. Equation (6.2) gives that $\log (1-z)=f+h$ and $h(T)$ is bounded. Hence it follows from general functional calculus theory [13, Cor. 1.2.3] that 
$\log (I-T)=f(T)+h(T)$ and that the domains of $\log (I-T)$ and $f(T)$ coincide. For the partial sums of $f$ and $\log (1-z)$ we obtain

$$
\sum_{k=0}^{n} \frac{z^{k}}{k+1}=\sum_{k=1}^{n} \frac{z^{k}}{k}+\left(1-\sum_{k=1}^{n} \frac{z^{k}}{k(k+1)}\right),
$$

which yields

$$
\sum_{k=0}^{n} \frac{T^{k}}{k+1} x=\sum_{k=1}^{n} \frac{T^{k} x}{k}+\left(x-\sum_{k=1}^{n} \frac{T^{k} x}{k(k+1)}\right)
$$

for each $n \geq 1$. Since the rightmost series converges in norm, the left-hand series converges (weakly) if and only if the middle one does. So all assertions follow directly from Theorem 5.6 .

Finally, we answer a question from [1, p. 23].

THEOREM 6.3. Let $T$ be a power-bounded operator on a Banach space $X$ such that $\operatorname{ran}(I-T)$ is dense in $X$ and $1 \in \sigma(T)$. Then the inclusion

$$
\bigcup_{s>0} \operatorname{ran}(I-T)^{s} \subset \operatorname{dom}(\log (I-T))
$$

is strict.

The inclusion (6.3) was proved in [11]. For $T$ being a unitary operator on a Hilbert space, the inclusion was shown to be strict in [1, and it was asked there whether it remains strict under the hypotheses of Theorem 6.3. Note that, together with the characterization $H_{T}=\log (I-T)$, the theorem shows that for a "generic" element in $\operatorname{dom}(I-T)$ the convergence of the ergodic averages does not happen with a polynomial rate.

Proof of Theorem 6.3. We employ the sectorial functional calculus sketched in Section 3. Let $A:=I-T$ and $B:=\log A$. We know that $A$ is an injective bounded sectorial operator. For every $\operatorname{Re} s>0$ and $n \in \mathbb{N}$ the function $z^{s}(\log z)^{n}$ lies in the basic function class $\mathcal{E}_{0}\left(S_{\pi}\right)$, i.e., is holomorphic on the sector $S_{\pi}$ and vanishes at zero with a polynomial rate. Since $\left(z^{s}\right)(A)=$ $A^{s}$ is bounded, functional calculus theory [13, Thm. 1.3.2.c] yields

$$
B^{n} A^{s}=(\log A)^{n} A^{s}=\left(z^{s}(\log z)^{n}\right)(A) \in \mathcal{L}(X) .
$$

This implies that $\operatorname{ran} A^{s} \subset \operatorname{dom} B^{n}$ for all $\operatorname{Re} s>0$ and $n \in \mathbb{N}$. On the other hand, suppose that one has equality

$$
\bigcup_{s>0} \operatorname{ran} A^{s}=\operatorname{dom}(\log A)=\operatorname{dom}(B) \text {. }
$$

Then in particular $\operatorname{dom}(B)=\operatorname{dom}\left(B^{2}\right)$. Since the operator $B=\log A$ always has non-empty resolvent set (Nollau's theorem [13, Lemma 3.5.1]), such an identity is only possible if $B$ is a bounded operator. But if $B=\log A$ is bounded then $I-T=A=e^{\log A}$ is invertible, whence $1 \notin \sigma(T)$. 
For the proof of Theorem 6.3 one can also use Remark 5.7 to deduce that $1 \notin \sigma(T)$. However, the proof given here works even under the weaker assumption $\operatorname{fix}(T)=0$ in place of $\overline{\operatorname{ran}}(I-T)=X$.

7. Final remarks. 1) On a conceptual level, the main difference between our approach and the one in [1] and [8] lies in the fact that we use $\|\cdot\|_{A_{+}^{1}(\mathbb{D})}$-estimates instead of $\|\cdot\|_{\infty}$-estimates in our function theory. Using the uniform norm restricts the results to operators that are essentially multiplication operators, e.g., normal contractions on Hilbert spaces. However, the transference principle formulated in Section 2 shows that the $\mathrm{A}_{+}^{1}(\mathbb{D})$ norm is exactly the right one when one aims at general results.

2) Our results are far from comprehensive. It is likely that with the same techniques one can obtain results for functions $f$ that are "higher-order" admissible in the sense that $-1 / f=\sum_{k} \beta_{k} z^{k}$ such that for some $N \in \mathbb{N}$, $\beta_{k} \leq 0$ for $0 \leq k \leq N-1$ and $\beta_{k} \geq 0$ for all $k \geq N$. Also, one would expect positive results when the condition $(1-z) f(z) \in \mathrm{A}_{+}^{1}(\mathbb{D})$ is replaced by $(1-z)^{M} f(z) \in \mathrm{A}_{+}^{1}(\mathbb{D})$, for some $M \in \mathbb{N}$.

3) The following function-theoretic problem turned out to be fundamental for our discussion. Suppose that $\alpha=\left(\alpha_{0}, \alpha_{1}, \ldots\right)$ is a sequence with $\alpha_{n} \searrow 0$ and $\alpha_{0}=1$. Define $f(z)=\widehat{\alpha}(z)=\sum_{k=0}^{\infty} \alpha_{n} z^{n}$ for $z \in \mathbb{D}$. Is it always true that $1 / f \in \mathrm{A}_{+}^{1}(\mathbb{D})$ ? If not, what conditions ensure that? Kaluza's theorem yields just a sufficient criterion, but it is far from necessary.

4) It is of interest to understand relations between analytic properties of the semigroup of fractional powers $\left((I-T)^{s}\right)_{s \geq 0}$ and its generator $G=$ $\log (I-T)$. In view of a special structure of the semigroup one might expect to find relations different from those following just from general semigroup theory.

Such relations are not straightforward. Recall an important assertion in semigroup theory saying that if $I-T$ is sectorial with angle less than $\pi / 2$, i.e., a Ritt type operator, if and only if $\left(e^{s(I-T)}\right)_{s \geq 0}$ is a sectorially bounded holomorphic (in fact entire) semigroup. This property does not transfer to the semigroup of fractional powers. Indeed, consider the Riemann-Liouville semigroup $\left(V^{s}\right)_{s \geq 0}$ on $L_{p}(0,1), 1 \leq p<\infty$, defined by

$$
\left(V^{s} f\right)(r)=\frac{1}{\Gamma(s)} \int_{0}^{r}(r-t)^{s-1} f(t) d t, \quad f \in L_{p}(0,1) .
$$

Recall that, while the semigroup $\left(V^{s}\right)_{s \geq 0}$ is bounded and extends holomorphically to the right half-plane (see e.g. [13, Chapter 3]), its generator $\log V$ is not a Ritt type operator (see [16, Chapter XXIII, 6]), so that $\left(V^{s}\right)_{s \geq 0}$ is not a sectorially bounded holomorphic semigroup. Recall also that by [24, 
Theorem] the operator $I-V^{s}$ is of Ritt type for every $s \in(0,1)$. For fixed $s_{0} \in(0,1)$ consider $T=I-V^{s_{0}}$. Then $(I-T)^{s}=V^{s_{0} s}, s \geq 0$, so that the generator $G$ of $\left(V^{s_{0} s}\right)_{s \geq 0}$ is given by

$$
G=\log V^{s_{0}}=s_{0} \log V
$$

and $\left((I-T)^{s}\right)_{s \geq 0}$ is not sectorially bounded.

On the other hand, if $I-T=(I-V)^{s}, s \in(0,1)$, then $I-T$ is a Ritt type operator by [12, Theorem 1.3]. At the same time, $\left((I-T)^{s}\right)_{s \geq 0}$ is not bounded on $L_{p}(0,1)$ unless $p=2$ (see [25, Theorem 1.1]).

5) Another question concerns the behavior of $\left((I-T)^{z}\right)_{\operatorname{Re} z>0}$ in the right half-plane. What conditions on $T$, apart from power-boundedness, guarantee that

$$
\int_{\mathbb{R}} \frac{\log _{+}\left\|(I-T)^{1+i t}\right\|}{1+t^{2}} d t<\infty ?
$$

Obtaining such conditions would open the door for applications of potential theory, e.g. Poisson integrals, to the study of $\left((I-T)^{z}\right)_{\operatorname{Re} z>0}$. (A similar question in a more general situation was asked by T. Ransford in [27].)

Acknowledgements. This work was initiated while the first author held a three-months fellowship of the EU-Marie Curie Project "TODEQ". The second author was partially supported by an MNiSZW grant No. N201384834. He would also like to thank the Functional Analysis Group of the Delft University of Technology for hospitality and nice working atmosphere in which this work was finished.

Both authors would like to thank M. Lin for making his preprints available to them prior to their publication.

\section{References}

[1] I. Assani and M. Lin, On the one-sided ergodic Hilbert transform, in: Ergodic Theory and Related Fields, Contemp. Math. 430, Amer. Math. Soc., Providence, RI, 2007, 21-39.

[2] C. Badea and V. Müller, On weak orbits of operators, Topology Appl. 156 (2009), 1381-1385.

[3] P. Butzer and U. Westphal, The mean ergodic theorem and saturation, Indiana Univ. Math. J. 20 (1971), 1163-1174.

[4] J. Campbell, Spectral analysis of the ergodic Hilbert transform, ibid. 35 (1986), 379-390.

[5] G. Cohen, C. Cuny and M. Lin, The one-sided ergodic Hilbert transform in Banach spaces, Studia Math. 196 (2010), 251-263.

[6] G. Cohen and M. Lin, Laws of large numbers with rates and the one-sided ergodic Hilbert transform, Illinois J. Math. 47 (2003), 997-1031. 
[7] G. Cohen and M. Lin, Extensions of the Menchoff-Rademacher theorem with applications to ergodic theory, in: Probability in Mathematics, Israel J. Math. 148 (2005), $41-86$.

[8] —, 一, The one-sided ergodic Hilbert transform of normal contractions, in: Characteristic Functions, Scattering Functions and Transfer Functions-the Moshe Livsic Memorial Volume, Birkhäuser, Basel, 2009, 77-98.

[9] C. Cuny, Pointwise ergodic theorems with rate and application to limit theorems for stationary processes, preprint.

[10] C. Cuny and M. Lin, Pointwise ergodic theorems with rate and application to the CLT for Markov chains, Ann. Inst. H. Poincaré Probab. Statist., to appear.

[11] Y. Derriennic and M. Lin, Fractional Poisson equations and ergodic theorems for fractional coboundaries, Israel J. Math. 123 (2001), 93-130.

[12] N. Dungey, Subordinated discrete semigroups of operators, preprint.

[13] M. Haase, The Functional Calculus for Sectorial Operators, Oper. Theory Adv. Appl. 169, Birkhäuser, Basel, 2006.

[14] P. R. Halmos, A non-homogeneous ergodic theorem, Trans. Amer. Math. Soc. 66 (1949), 284-288.

[15] G. H. Hardy, Divergent Series, Clarendon Press, Oxford, 1949.

[16] E. Hille and R. Phillips, Functional Analysis and Semi-Groups, Colloq. Publ. 31, Amer. Math. Soc., 1957.

[17] F. Hirsch, Domaines d'opérateurs représentés commes intégrales des résolvantes, J. Funct. Anal. 23 (1976), 199-217.

[18] S. Izumi, A non-homogeneous ergodic theorem, Proc. Imp. Acad. Tokyo 15 (1939), 189-192.

[19] S. Kakutani and K. Petersen, The speed of convergence in the ergodic theorem, Monatsh. Math. 91 (1981), 11-18.

[20] Th. Kaluza, Über die Koeffizienten reziproker Potenzreihen, Math. Z. 28 (1928), 161-170.

[21] D. G. Kendall, Renewal sequences and their arithmetics, in: Lecture Notes in Math. 31, Springer, 1967, 147-175.

[22] -, Delphic semi-groups, infinitely divisible regenerative phenomena, and the arithmetic of p-functions, Z. Wahrsch. Verw. Gebiete 9 (1968), 163-195.

[23] U. Krengel, Ergodic Theorems, with a supplement by Antoine Brunel, de Gruyter Stud. Math. 6, de Gruyter, Berlin, 1985.

[24] Yu. Lyubich, The single-point spectrum operators satisfying Ritt's resolvent condition, Studia Math. 145 (2001), 135-142.

[25] A. Montes-Rodríguez, H. Sánchez-Álvarez and J. Zemánek, Uniform Abel-Kreiss boundedness and the extremal behavior of the Volterra operator, Proc. London Math. Soc. 91 (2005), 761-788.

[26] V. Nollau, Über den Logarithmus abgeschlossener Operatoren in Banachschen Räumen, Acta Sci. Math. (Szeged) 30 (1969), 161-174.

[27] T. Ransford, Norm inequalities for holomorphic semigroups, Israel J. Math. 97 (1997), 157-173.

[28] R. Schilling, Subordination in the sense of Bochner and a related functional calculus, J. Austral. Math. Soc. 64 (1998), 368-396.

[29] G. Szegö, Bemerkungen zu einer Arbeit von Herrn Fejér über die Legendreschen Polynome, Math. Z. 25 (1926), 172-187.

[30] U. Westphal, A generalized version of the Abelian mean ergodic theorem with rates for semigroup operators and fractional powers of infinitesimal generators, Results Math. 34 (1998), 381-394. 
[31] A. Zygmund, Trigonometric Series, Vols. I, II, 3rd ed., Cambridge Univ. Press, 2002.

Markus Haase

Yuri Tomilov

Delft Institute of Applied Mathematics Faculty of Mathematics and Computer Science

Delft University of Technology

P.O. Box 5031

Nicolas Copernicus University

2600 GA Delft, The Netherlands

Chopina 12/18

E-mail: m.h.a.haase@tudelft.nl

87-100 Toruń, Poland

E-mail: tomilov@mat.uni.torun.pl

and

Institute of Mathematics

Polish Academy of Sciences

Śniadeckich 8

00-956 Warszawa, Poland

Received May 6, 2009

(6619) 